\title{
Mean platelet volume: a new predictor of ischaemic stroke risk in patients with nonvalvular atrial fibrillation
}

\author{
Meifang Zheng ${ }^{1,2}$, Shean Chen ${ }^{1,2}$, Ye Zhu ${ }^{1,2}$ and Xiang Gu ${ }^{1,2^{*}}$ (D)
}

\begin{abstract}
Background: Mean platelet volume (MPV) has been identified as an individual risk factor for stroke and thrombosis. Concurrently, ischaemic stroke caused by nonvalvular atrial fibrillation (AF) has attracted increasing attention. The aim of this study was to investigate the association between MPV and the risk of ischaemic stroke in AF patients not receiving anticoagulant therapy.

Methods: A total of 370 patients with nonvalvular AF were enrolled. Patients were divided into a control group and a stroke group according to the presence of ischaemic stroke.

Results: The MPV level and CHA2DS2-VASc scores of the stroke group were higher than those of the control group (all $p<0.001$ ). The ischaemic stroke event rates were significantly increased in the highest MPV tertile when compared to the lowest MPV tertile (56.9\% vs. 30.3\%, $p<0.001)$. Multivariate logistic regression analysis showed that CHA2DS2-VASC, MPV and D-dimer (D2) were predictors of ischaemic stroke [all $p<0.05$ ]. The receiver operating characteristic (ROC) curve analysis indicated that an MPV value of $11.65 \mathrm{fL}$ could predict ischaemic stroke with a sensitivity of $67.3 \%$ and specificity of $58.5 \%$, while a CHA2DS2-VASc score cutoff value 3.5 had a sensitivity of $52.1 \%$ and specificity of $87.3 \%$. The predictive value of the combined model of CHA2DS2-VASC+MPV was higher than others (comparison calculated by using MedCalc software). The sensitivity of the CHA2DS2-VASc score combined with MPV for predicting ischaemic stroke was $72.1 \%$, and the specificity was $81.5 \%$.
\end{abstract}

Conclusions: MPV could be a new predictor of ischaemic stroke risk in patients with AF. Moreover, the CHA2D2S2VASC combined with MPV can improve predictive value with higher sensitivity and it could be a powerful tool for stratifying patients in terms of ischaemic stroke risk.

Keywords: Atrial fibrillation, Mean platelet volume, Ischaemic stroke, CHA2DS2-VASc score

\section{Background}

Accurate ischaemic stroke risk stratification in atrial fibrillation (AF) patients is an important aspect of AF management. The CHA2DS2-VASc (congestive heart failure, hypertension, age $\geq 75$ years, diabetes mellitus, stroke or transient ischaemic stroke (TIA) or other embolic

\footnotetext{
* Correspondence: guxiang@yzu.edu.cn

'Clinical Medical College, Yangzhou University, Yangzhou, Jiangsu, China ${ }^{2}$ Department of Cardiology, Northern Jiangsu People's Hospital, No. 98, Nantong West Road, Yangzhou 225001, Jiangsu, China
}

events, vascular disease, age 65 to 74 years, sex category) score is the most widely used AF-related ischaemic stroke risk stratification tool in the world today. It was recommended as the only risk stratification tool for ischaemic stroke in patients with AF by the 2016 ESC Guidelines [1] and the 2019 AHA/ACC/HRS Guidelines [2].

Studies have shown that thromboembolic events in patients with $\mathrm{AF}$ are associated with the presence of a prothrombotic state (PTS) [3]. PTS refers to a pathological

(c) The Author(s). 2020 Open Access This article is licensed under a Creative Commons Attribution 4.0 International License, which permits use, sharing, adaptation, distribution and reproduction in any medium or format, as long as you give appropriate credit to the original author(s) and the source, provide a link to the Creative Commons licence, and indicate if changes were made. The images or other third party material in this article are included in the article's Creative Commons licence, unless indicated otherwise in a credit line to the material. If material is not included in the article's Creative Commons licence and your intended use is not permitted by statutory regulation or exceeds the permitted use, you will need to obtain permission directly from the copyright holder. To view a copy of this licence, visit http://creativecommons.org/licenses/by/4.0/ The Creative Commons Public Domain Dedication waiver (http://creativecommons.org/publicdomain/zero/1.0/) applies to the data made available in this article, unless otherwise stated in a credit line to the data. 
condition caused by various factors, such as haemostasis, coagulation and anticoagulant system abnormalities. Platelet activation is a component of PTS, and markers of platelet activation include mean platelet volume (MPV), soluble P-selectin, platelet factor 4 , and glycoprotein IIb/IIIa [4]. MPV is an accurate marker of platelet size and is a routine item in the analysis of whole blood counts. It is more convenient and economical to measure than other platelet activation markers. Studies have shown that a high MPV level was independently associated with thrombosis [5, 6]. Furthermore, some studies have shown that MPV can be used to predict ischaemic stroke risk in patients with AF [7-9]. It was especially suitable for patients with AF with a low to intermediate traditional thromboembolic risk (CHADS2 score $<2$, congestive heart failure, hypertension, age $\geq 75$ years, diabetes mellitus, stroke), which has a complementary role in the prevention of ischaemic stroke in AF patients [10].

In the previous study, the researchers did not stratify AF patients with or without anticoagulant treatment. However, due to the differences in drug-related genes and the lack of regular monitoring of the international normalized ratio in the East Asian region, especially in China, the anticoagulation intensity among patients in these studies may differ. In this study, we focused on patients with AF who were not treated with anticoagulant therapy, which can reduce the error caused by different strengths of anticoagulation. We explored the risk factors of AF-related ischaemic stroke in the Chinese population and analysed the value of MPV, as well as the CHA2DS2-VASc score combined with MPV, in predicting AF-related ischaemic stroke risk to provide effective guidance for the prevention and management of AFrelated ischaemic stroke.

\section{Methods}

\section{Study population}

A total of 370 patients with nonvalvular AF who underwent MPV measurement at our hospital between January 2017 and December 2018 were included in this study. Verbal informed consent were obtained from all involved participants, and the study was conducted according to the Helsinki Declaration. The study was approved by the ethics committee of Northern Jiangsu People's Hospital. We included patients between the ages of 18 and 80 years who were not using any anticoagulation drugs. Patients with valvular heart disease, congenital heart disease, thyroid disease, malignant tumours, autoimmune disease, severe infection, blood system disease, connective tissue disease, rheumatic activity, pulmonary embolism, deep vein thrombosis, severe liver and kidney dysfunction, electrolyte imbalance, history of traumatic surgery or acute myocardial infarction within the past 3 months combined with other types of arrhythmias, were excluded. The included patients were followed up until December 31, 2019, they were divided into two groups according to the presence of acute ischaemic stroke during the follow-up period. The diagnosis of acute ischaemic stroke conformed to the 2014 "Guidelines for the Diagnosis and Treatment of Acute Ischaemic Stroke in China" [11]. The diagnostic criteria were as follows: (1) acute onset; (2) regional neurologic impairment; (3) the duration of symptoms or signs is not limited (when imaging shows that there is a responsible ischaemic lesion) or lasts for more than $24 \mathrm{~h}$ (when imaging shows that there is no responsible lesion); (4) nonvascular causes have been excluded; and (5) cerebral CT/MRI excluded cerebral haemorrhage. Besides, all patients included in the stroke group were excluded from the possibility of stroke from other causes, like patent foramen ovale tested by echocardiography, prominent carotid artery stenosis tested by carotid duplex ultrasound, and cerebral arterial stenosis tested by TCD (intracranial dopley). The possibility of cardioembolism was considered by doctors to be the greatest with the evidence of AF in ECG or Holter. The control group consisted of 205 patients with AF, and the stroke group consisted of 165 patients with AF-related ischaemic stroke. All patients in the stroke group were recorded with atrial fibrillation by electrocardiogram prior to stroke.

\section{Data collection}

The clinical data of all patients were collected at the time of the first diagnosis of AF, including basic demographic characteristics, previous disease history, basic medication use, MPV and other items included within the whole blood count, fibrinogen (FIB), D-dimer (D2), creatinine $(\mathrm{Cr})$ and uric acid (UA), left atrial diameter (LAD) and left ventricular ejection fraction (LVEF). All the blood test results were from the first venous blood test results after admission and prior to any treatment. The CHA2DS2-VASc scores of all patients were calculated. The neutrophil-lymphocyte ratio (NLR) is the neutrophil count divided by the lymphocyte count. Vascular disease refers to myocardial infarction, complex aortic plaque, and peripheral artery disease including revascularization, amputation because of peripheral artery disease, or angiographic evidence of peripheral artery disease.

\section{Statistical analysis}

Data were analysed with SPSS software version 20.0 for Windows (SPSS Inc., Chicago, Illinois, USA). Continuous variables with a normal distribution were described by the mean \pm standard deviation, and continuous variables without a normal distribution were described by 
quartile. The categorical variables were summarized as frequencies and percentages. Categorical variables were compared by the chi-squared test or Fisher's exact test. Comparisons between two continuous variables with normal distribution were carried out with the independent samples Student's t-test, and comparisons between two continuous variables without normal distributions were carried out with the Mann-Whitney U test. Univariate and multivariate binary logistic regression analysis with the backward likelihood ratio method were employed to determine the predictors of ischaemic stroke. Receiver operating characteristic (ROC) curve analysis was performed to determine the sensitivity and specificity with a $95 \%$ confidence interval (CI) for the MPV and CHA2DS2-VASc at cutoff values calculated by the Youden index. Medcalc software was used to compare different ROC curves. A two-tailed $p$ value of less than 0.05 was considered statistically significant.

\section{Results}

\section{Clinical characteristics}

A total of 370 patients with nonvalvular AF were enrolled, including 206 males (55.7\%), and the mean age of all patients was $68.16 \pm 8.75$ years. A total of 205 patients were included in the control group, and 165 patients were included in the stroke group. The clinical characteristics of the two groups are shown in Table 1. The two groups were significantly different in terms of age as well as in history of heart failure, hypertension, diabetes, ischaemic stroke or transient ischaemic stroke and vascular disease $(p<0.05$ for all variables). The blood test and echocardiography results of the two groups are shown in Table 1. The MPV level of the stroke group was higher than that of the control group $(12.08 \pm 1.05$ vs. $11.42 \pm 1.08 \mathrm{fL}, \quad p<0.001)$. The CHA2DS2-VASc score of the stroke group was $2.07 \pm 1.31$, while the score of the control group was $2.04 \pm 1.31$, and the difference was statistically significant $(p<0.001)$.

\section{Predictors of Ischaemic stroke}

Univariate binary logistic regression analysis showed that WBC, MPV, D2, Cr and the CHA2DS2-VASc score were all risk factors (all $p<0.01$ ) (Table 2), whereas multivariate binary logistic regression analysis with the backward likelihood ratio method showed that only the CHA2DS2-VASc score $(\mathrm{OR}=2.154$; 95\% CI, 1.739$2.668 ; p<0.001)$, MPV $(\mathrm{OR}=1.962 ; 95 \% \mathrm{CI}, 1.493-$ 2.579; $p<0.001)$ and $\mathrm{D} 2(\mathrm{OR}=1.80 ; 95 \% \mathrm{CI}, 1.13-2.84$; $p=0.012)$ were risk factors for ischaemic stroke in patients with AF (Table 2). (Cr was not further analysed because the $\mathrm{Cr}$ OR was 0.98 , which means a low correlation between $\mathrm{Cr}$ and ischaemic stroke).

\section{Subgroup analyses by MPV and CHA2DS2-VASc score}

Patients were stratified into tertiles according to MPV $(<11.2 \mathrm{fL}, 11.2-12.2 \mathrm{fL}$ and $\geq 12.2 \mathrm{fL})$, and a subgroup analysis was performed using the $\mathrm{x} 2$ test. The results showed that the ischaemic stroke event rates increased significantly in the highest MPV tertile group when compared to the lowest MPV tertile group $(56.9 \%$ vs. $30.3 \%, p<0.001$ ) (Fig. 1a).

Furthermore, subgroup analyses stratified according to the CHA2DS2-VASc score were performed to further verify the predictive power of the CHA2DS2-VASc score. The results showed that the incidence of ischaemic stroke gradually increased with increasing CHA2DS2-VASc score; in the low-risk group $($ CHA2DS2-VASc $=0)$, the middle-risk group $($ CHA2DS2-VASc $=1)$ and the high-risk group (CHA2DS2-VASc $\geq 2)$ the incidence was $0,26.3$, and $53.3 \%$, respectively. The difference was statistically significant. $(p<0.05)$ (Fig. 1a).

\section{The predictive value of risk factors}

The predictive value of risk factors for predicting ischaemic stroke events in patients with AF was evaluated by ROC analysis. The area under the curve (AUC) values of MPV, D-dimer and CHA2DS2-VASc were 0.664, 0.668 and 0.761 , respectively (Table 3 , Fig. $1 \mathrm{~b}$ ). The results of the ROC analysis indicated a cutoff value of $11.65 \mathrm{fL}$ for MPV with $67.3 \%$ sensitivity and $58.5 \%$ specificity $(p<$ 0.001 ) for the prediction of ischaemic stroke, while the CHA2DS2-VASc score cutoff value of 3.5 had a sensitivity of $52.1 \%$ and specificity of $87.3 \%$. In addition, the cutoff value of D-dimer was $0.485 \mathrm{mg} / \mathrm{L}$; the sensitivity was $68.5 \%$, and the specificity was $63.8 \%$.

\section{The predictive ability of combined predictive models}

To assess the predictive ability of CHA2DS2-VASc combined with MPV, logistic regression analysis was conducted with group as the dependent variable and CHA2DS2-VASc and MPV as independent variables. The probability value $(\mathrm{P})$ was calculated by SPSS software during regression analysis. The probability value (P) was the test variable, and the group was the state variable in the ROC analysis. The area under the curve (AUC) value for the CHA2DS2-VASc combined with MPV model was 0.812 , and the cutoff value, which was calculated by the Youden index, was 0.487 , with $72.1 \%$ sensitivity and $81.5 \%$ specificity $(p<0.001)$ for the prediction of ischaemic stroke (Fig. 1b). Using the same method, we obtained an CHA2DS2VASc+D2 AUC value of 0.783 , a sensitivity of $69.1 \%$ and a specificity of $78.4 \%(p<0.001)$ (Table 3). Medcalc software was used to compare various ROC curves, and the results showed that the CHA2DS2VASc+MPV AUC value was higher than that of 
Table 1 Baseline characteristics of the two groups

\begin{tabular}{|c|c|c|c|c|}
\hline Variables & Control group $(n=205)$ & Stroke group $(n=165)$ & t/Z /X2 value & $p$ value \\
\hline Male (\%) & $117(57.1)$ & $89(53.9)$ & 0.364 & 0.546 \\
\hline Age (years old) & $65.40 \pm 9.48$ & $71.58 \pm 6.24$ & 5.087 & $<0.001^{* * *}$ \\
\hline $\mathrm{BMI}(\mathrm{kg} / \mathrm{m} 2)$ & $24.34 \pm 3.12$ & $24.48 \pm 3.64$ & 0.802 & 0.690 \\
\hline Smoke (\%) & $41(20.0)$ & $34(20.6)$ & 0.021 & 0.885 \\
\hline Drink (\%) & $27(13.2)$ & $29(17.6)$ & 1.381 & 0.240 \\
\hline Heart failure (\%) & $5(2.4)$ & $14(8.5)$ & 6.859 & $0.009^{* *}$ \\
\hline Hypertension (\%) & $105(51.2)$ & $120(72.7)$ & 17.745 & $<0.001^{* * *}$ \\
\hline Diabetes (\%)) & $22(10.7)$ & $38(23.0)$ & 10.177 & $0.001^{* *}$ \\
\hline Stroke/TIA (\%) & $13(6.3)$ & $49(29.7)$ & 35.750 & $<0.001^{* * *}$ \\
\hline Vascular disease (\%) & $16(7.8)$ & $24(14.5)$ & 4.308 & $0.038^{* *}$ \\
\hline COPD (\%) & $3(1.5)$ & $3(1.8)$ & 0.072 & 0.789 \\
\hline Gout (\%) & $4(2.0)$ & $1(0.6)$ & 1.352 & 0.386 \\
\hline CCB (\%) & $40(19.5)$ & $32(19.4)$ & 0.001 & 0.977 \\
\hline Statins (\%) & $11(5.4)$ & $12(7.3)$ & 0.570 & 0.450 \\
\hline Diuretic (\%) & $8(3.9)$ & $2(1.2)$ & 2.516 & 0.195 \\
\hline ACEI/ARB (\%) & $49(23.9)$ & $26(15.8)$ & 3.753 & 0.053 \\
\hline Digoxin (\%) & $4(2.0)$ & $4(2.4)$ & 0.097 & 0.756 \\
\hline Antiplatelet drugs (\%) & $23(11.2)$ & $30(18.2)$ & 3.611 & 0.057 \\
\hline$\beta$-blocker (\%) & $34(16.6)$ & $16(9.7)$ & 3.712 & 0.054 \\
\hline $\mathrm{RBC}\left(\times 10^{12} / \mathrm{L}\right)$ & $4.54 \pm 0.55$ & $4.59 \pm 0.52$ & 0.848 & 0.397 \\
\hline HGB (g/L) & $139.76 \pm 15.79$ & $139.27 \pm 15.83$ & 0.295 & 0.768 \\
\hline HCT (\%) & $41.72 \pm 4.55$ & $41.57 \pm 4.29$ & 0.325 & 0.746 \\
\hline $\mathrm{MCHC}(\mathrm{g} / \mathrm{L})$ & $3.35 \pm 0.128$ & $3.34 \pm 0.135$ & 0.140 & 0.889 \\
\hline $\mathrm{MCV}(\mathrm{fl})$ & $91.95 \pm 6.18$ & $90.73 \pm 5.05$ & 2.030 & $0.043^{*}$ \\
\hline RDW-CV (\%) & $13.42 \pm 2.37$ & $13.67 \pm 2.25$ & 1.032 & 0.303 \\
\hline WBC $\left(\times 10^{9} / L\right)$ & $6.45 \pm 2.01$ & $7.22 \pm 2.50$ & 3.302 & $0.001^{* *}$ \\
\hline $\operatorname{PLT}\left(\times 10^{9} / \mathrm{L}\right)$ & $176.01 \pm 51.73$ & $171.38 \pm 53.15$ & 0.845 & 0.399 \\
\hline MPV (fl) & $11.42 \pm 1.08$ & $12.08 \pm 1.05$ & 5.948 & $<0.001^{* * *}$ \\
\hline PDW (\%) & $14.84 \pm 3.51$ & $15.91 \pm 2.94$ & 3.116 & $0.002^{* *}$ \\
\hline $\mathrm{FIB}(\mathrm{g} / \mathrm{L})$ & $2.97 \pm 0.91$ & $2.79 \pm 0.70$ & 1.916 & 0.056 \\
\hline D2 (mg/L) & $0.36(0.24-0.81)$ & $0.71(0.39-1.35)$ & 5.269 & $<0.001^{* * *}$ \\
\hline $\mathrm{UA}(\mu \mathrm{mol} / \mathrm{L})$ & $360.91 \pm 110.73$ & $349.50 \pm 103.19$ & 1.011 & 0.313 \\
\hline $\mathrm{Cr}(\mu \mathrm{mol} / \mathrm{L})$ & $82.37 \pm 21.37$ & $73.29 \pm 23.09$ & 3.899 & $<0.001^{* * *}$ \\
\hline LAD (mm) & $37.83 \pm 6.27$ & $40.2 \pm 5.90$ & 3.468 & $0.001^{* *}$ \\
\hline LVEF (\%) & $57.80 \pm 7.45$ & $57.04 \pm 7.20$ & 0.935 & 0.350 \\
\hline NLR & $2.33(1.71-3.58)$ & $3.11(1.94-5.44)$ & 4.017 & $<0.001^{* * *}$ \\
\hline CHADS2 & $0.94 \pm 0.93$ & $2.07 \pm 1.31$ & 27.206 & $<0.001^{* * *}$ \\
\hline CHA2DS2-VASC & $2.04 \pm 1.31$ & $3.54 \pm 1.56$ & 9.896 & $<0.001^{* * *}$ \\
\hline
\end{tabular}

CHA2DS2-VASc or MPV alone, and the difference was statistically significant $(p<0.05)$ (Table 4$)$. The predictive value of the combined CHA2DS2$\mathrm{VASc}+\mathrm{MPV}+\mathrm{D} 2$ model was higher than that of CHA2DS2-VASc+D2 $(p=0.034)$ but was similar to that of CHA2DS2-VASc+MPV $(p=0.264)$. In addition, there was no significant difference between the CHA2DS2-VASc+MPV and CHA2DS2-VASc+D2 models $(p=0.174)$; therefore, the combination of the CHA2DS2-VASc score plus MPV had higher sensitivity and specificity and could significantly improve the prediction of ischaemic stroke in patients with AF. 
Table 2 Risk factors for ischemic stroke analyzed by logistic regression

\begin{tabular}{|c|c|c|c|c|c|c|c|c|}
\hline \multirow[t]{2}{*}{ Variables } & \multicolumn{4}{|l|}{ univariate } & \multicolumn{4}{|c|}{ multivariate } \\
\hline & $p$ value & OR & $95 \% \mathrm{Cl}$ & & $p$ value & OR & $95 \% \mathrm{Cl}$ & \\
\hline WBC & $0.001^{* *}$ & 1.167 & 1.061 & 1.284 & 0.081 & 1.126 & 0.986 & 1.286 \\
\hline MPV & $<0.001^{* * *}$ & 1.783 & 1.450 & 2.191 & $<0.001^{* * *}$ & 1.962 & 1.493 & 2.579 \\
\hline D2 & $0.006^{* *}$ & 1.318 & 1.082 & 1.606 & $0.04^{*}$ & 1.230 & 1.009 & 1.498 \\
\hline $\mathrm{Cr}$ & $<0.001^{* * *}$ & 0.981 & 0.971 & 0.991 & $0.002^{* *}$ & 0.980 & 0.967 & 0.992 \\
\hline CHA2DS2-VASC & $<0.001^{* * *}$ & 2.029 & 1.710 & 2.408 & $<0.001^{* * *}$ & 2.154 & 1.739 & 2.668 \\
\hline
\end{tabular}

\section{Discussion}

The results suggest that MPV was a risk factor for ischaemic stroke in patients with nonvalvular AF. CHA2DS2-VASc combined with MPV can improve the predictive sensitivity of ischaemic stroke in patients with AF. To the best of our knowledge, this is the first study in China to report that MPV combined with CHA2DS2VASc scores can improve the predictive value of ischaemic stroke in AF patients.

MPV is a clinical indicator with a normal range of 7$11 \mathrm{fL}$, which can reflect changes in platelet activation or platelet production. In fact, larger platelets contain denser particles than smaller platelets; they can release more thromboxane A2 and beta-thrombin, and they express more glycoprotein IIb/IIIa receptors. That is, MPV levels are positively correlated with platelet activity and are associated with thrombosis. High MPV levels have been reported to be associated with a variety of cardiovascular and cerebrovascular-related embolic diseases [12-15]. A study published in the European Heart Journal article showed that elevated MPV levels were associated with an increased risk of deep venous thrombosis of the lower extremities and acute myocardial infarction, and increased MPV was considered a predictor of venous thromboembolism [16].

Platelet activity is significantly increased in patients with AF because of the presence of PTS; thus, MPV and other various platelet activity markers are increased [6, $15,16]$. The measurement of other markers (such as soluble P-selectin, platelet factor 4, and glycoprotein IIb/ IIIa) is complex, time-consuming, and costly and cannot be routinely applied to normal clinical work. MPV, a routine blood cell analysis index, is easy to obtain in the clinic and in the hospital, and its measurement is inexpensive and can be widely used in clinical work. Several studies have found that increased MPV is a risk factor for ischaemic stroke in patients with AF. Soon-Pyo Hong et al. analysed the risk of ischaemic stroke in 265 patients with AF undergoing ventricular rate or rhythm control and obtained high MPV levels $(\geq 7.85 \mathrm{fL})$, high CHADS2 scores ( $\geq 2$ points) and lack of ventricular rate control as risk factors for ischaemic stroke in a Cox proportional hazards regression model, independent of sex or anticoagulant therapy [7]. Nermin Bayar et al. divided

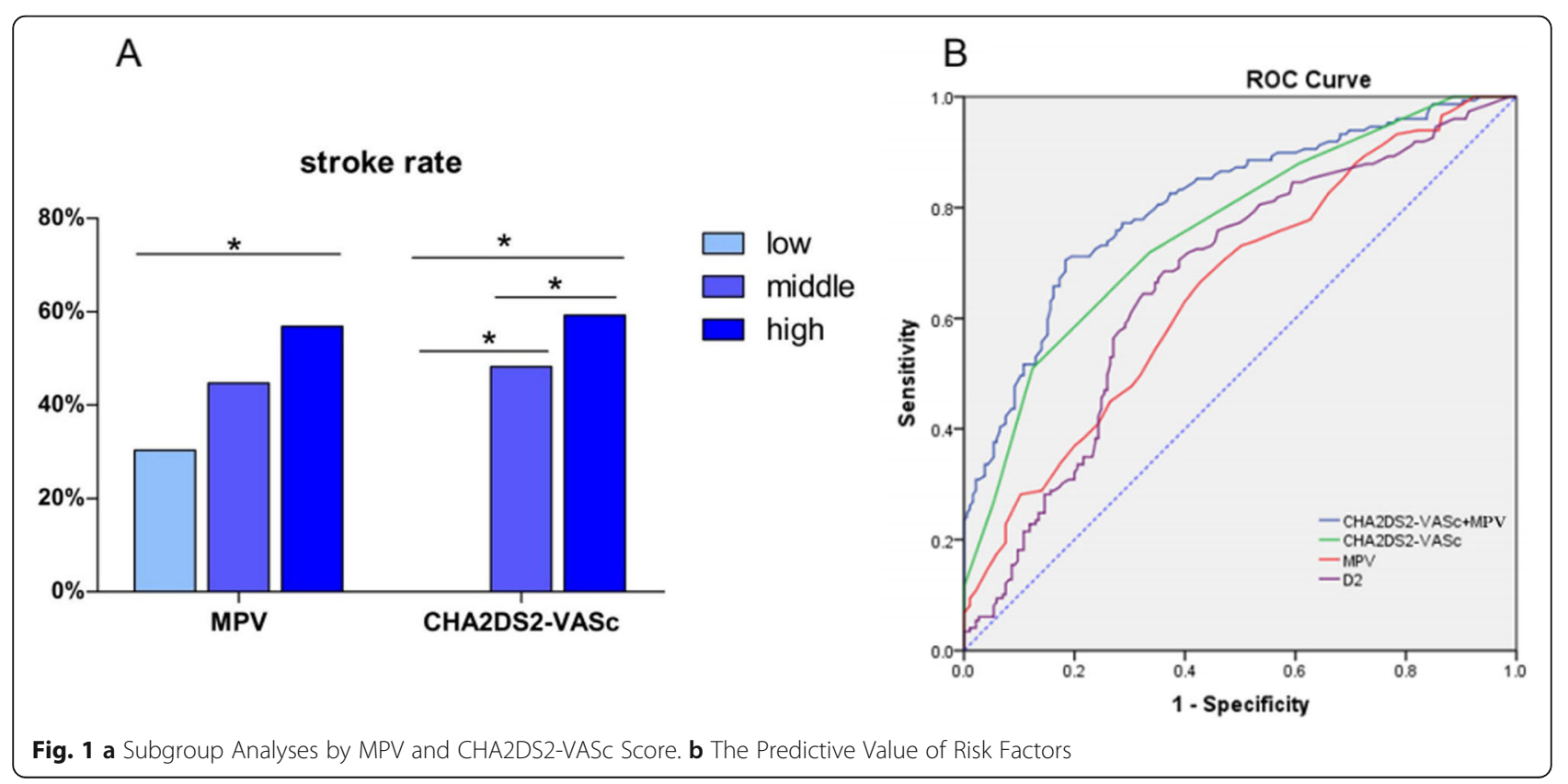


Table 3 The ROC analysis of risk factors

\begin{tabular}{|c|c|c|c|c|c|}
\hline Risk factors & $A \cup C$ & $p$ value & cut-off & Sensitivity (\%) & Specificity $(\%)$ \\
\hline MPV & 0.664 & $<0.001^{* * *}$ & 11.65 & 67.3 & 58.5 \\
\hline D2 & 0.668 & $<0.001^{* * *}$ & 0.485 & 68.5 & 63.8 \\
\hline CHA2DS2-VASC & 0.761 & $<0.001^{* * *}$ & 3.5 & 52.1 & 87.3 \\
\hline CHA2DS2-VASC+D2 & 0.783 & $<0.001^{* * *}$ & 0.472 & 69.1 & 78.4 \\
\hline CHA2DS2-VASC+MPV & 0.812 & $<0.001^{* * *}$ & 0.487 & 72.1 & 81.5 \\
\hline $\mathrm{CHA} 2 \mathrm{DS} 2-\mathrm{VASC}+\mathrm{MPV}+\mathrm{D} 2$ & 0.816 & $<0.001^{* * *}$ & 0.479 & 71.1 & 81.1 \\
\hline
\end{tabular}

90 patients with paroxysmal AF into a symptomatic group and an asymptomatic group based on the presence of ischaemic stroke or TIA. Statistical analysis showed no statistically significant differences between the two groups. The MPV value of the symptomatic group was higher than that of the asymptomatic group $(p<0.001)$. The ROC curve analysis showed that the cutoff value of MPV for predicting ischaemic stroke was $9.85 \mathrm{fL}$; the sensitivity was $87 \%$, and the specificity was $78 \%$ [8]. The results suggested that MPV can be used as a predictor of ischaemic stroke in patients with paroxysmal AF. A study published in 2017 of the relationship between antithrombin III combined with MPV and the risk of ischaemic stroke or left atrial thrombosis in patients with AF indicated that high MPV and lack of antithrombin III were risk factors for ischaemic stroke or left atrial thrombus in patients with AF [17].

The results of this study show that MPV was a risk factor for ischaemic stroke in patients with AF. For every additional unit of MPV, the odds of ischaemic stroke in AF patients increased 1.962 times. In addition, in the subgroup analysis, the higher the MPV value, the higher the proportion of patients with ischaemic stroke $(p<$ 0.001 ). These subgroup analyses further validated the relationship between MPV and the risk of ischaemic stroke at different levels. The ROC curve analysis showed that MPV was a predictor for ischaemic stroke in patients with AF, the AUC was 0.664, and the cutoff value was

Table 4 Comparison of different ROC curves

\begin{tabular}{lll}
\hline Different ROC curves & Z value & $p$ value \\
\hline MPV D2 & 0.296 & 0.767 \\
CHA2DS2-VASC D2 & 2.595 & $0.009^{* *}$ \\
CHA2DS2-VASC MPV & 2.531 & $0.011^{*}$ \\
CHA2DS2-VASC +MPV CHA2DS2-VAS & 3.328 & $<0.001^{* * *}$ \\
CHA2DS2-VASC +MPV MPV & 5.565 & $<0.001^{* * *}$ \\
CHA2DS2-VASC +D2 CHA2DS2-VASC & 2.576 & $0.010^{*}$ \\
CHA2DS2-VASC +D2 D2 & 3.777 & $<0.001^{* * *}$ \\
CHA2DS2-VASC +MPV CHA2DS2-VASC +D2 & 1.359 & 0.174 \\
CHA2DS2-VASC +MPV + D2 CHA2DS2-VASC +MPV & 1.118 & 0.264 \\
CHA2DS2-VASC +MPV + D2 CHA2DS2-VASC +D2 & 2.123 & $0.034^{*}$ \\
\hline
\end{tabular}

$11.65 \mathrm{fL}$, with $67.3 \%$ sensitivity and $58.5 \%$ specificity for the prediction of ischaemic stroke. This was similar to the results of the above studies abroad, but the cutoff value, AUC value, sensitivity and specificity obtained in this study were slightly different from those of other studies, which may be related to the different blood test methods and reagents, as well as the inclusion and exclusion criteria of the population. In addition, this study was a retrospective study with a relatively small sample size.

The CHA2DS2-VASc score is currently the most widely used AF-related ischaemic stroke risk stratification tool in the world. However, the predictive value of the CHA2DS2-VASc score is at a moderate level [2], and this study found that the AUC was 0.761 with a sensitivity of $52.1 \%$ and a specificity of $87.3 \%$ when using CHA2DS2-VASc alone to predict ischaemic stroke. However, the AUC was 0.812 , with a sensitivity for the CHA2DS2-VASc combined with MPV of $72.1 \%$, which is significantly higher than that of the CHA2D2S2-VASc score alone. It is beneficial to screen patients with $\mathrm{AF}$ and a low risk of ischaemic stroke so that timely secondary prevention can be initiated in more patients with increased ischaemic stroke risk to reduce the incidence of ischaemic stroke events.

\section{Limitations}

This study was a single-centre retrospective study. The sample size was limited. Due to regional factors and different detection methods, the results may be different from those conducted in other areas. Multi-centre largescale studies are needed to reduce this bias. In addition, the patients included in this study were all AF patients who were not receiving anticoagulant therapy. Therefore, we were unable to analyse whether anticoagulant therapy had an effect on biomarkers such as MPV and D-dimer.

\section{Conclusion}

This study concluded that MPV was a risk factor for ischaemic stroke in patients with AF. High MPV (cutoff value of $11.65 \mathrm{fL}$ ) can be used to predict ischaemic stroke risk in patients with AF. MPV could be a new predictor of ischaemic stroke risk in patients with AF. 
Moreover, CHA2DS2-VASc combined with MPV can improve the sensitivity of predicting ischaemic stroke risk, the combination of these two measures could be a powerful risk stratification tool for patients with AF. It will be helpful for identifying patients with low ischaemic stroke risk and increasing secondary prevention of ischaemic stroke in patients with AF.

\section{Abbreviations}

MPV: Mean platelet volume; AF: Atrial fibrillation; ROC: Receiver operating characteristic; TIA: Transient ischemic stroke; PTS: Prothrombotic state; FIB: Fibrinogen; D2: D-dimer; Cr: Creatinine; UA: Uric acid; LAD: Left atrial diameter; LVEF: Left ventricular ejection fraction; NLR: Neutrophil-lymphocyte ratio; Cl: Confidence interval; AUC: Area under the curve

\section{Acknowledgements}

The authors would like to appreciate the assistance of Dr. Zhengyu Bao, Dr. Lei Sun and Dr. Zhen Wang for their valuable advice on the management of the patient. We thank the editor and the reviewers whose comments/ suggestions helped improve and clarify this manuscript.

\section{Authors' contributions}

MFZ: Conceptualization, Investigation, Data curation, Methodology, Formal analysis, Validation, Writing-original draft, Writing-review \& editing, Visualization, together with SAC and YZ. XG: Conceptualization, Methodology, Validation, Formal analysis. All the authors were involved in the draft, revision and approval of the final version.

\section{Funding}

This work was supported by funds from science and technology department of Jiangsu province, China (No. BL2013022). Funding is mainly used for chronic follow-up and paper publication.

\section{Availability of data and materials}

The datasets analyzed during the current study are available from the corresponding author on reasonable request.

\section{Ethics approval and consent to participate}

Verbal informed consent were obtained from all involved participants and the study was performed in accordance with the Declaration of Helsinki and approved by the institutional ethical board of Northern Jiangsu Province People's Hospital, Jiangsu Province, China.

\section{Consent for publication}

Not applicable.

\section{Competing interests}

The authors have no conflicts of interest to declare.

Received: 12 March 2020 Accepted: 12 May 2020

Published online: 20 May 2020

\section{References}

1. Kirchhof P, Benussi S, Kotecha D, Ahlsson A, Atar D, Casadei B, Castella M, Diener HC, Heidbuchel H, Hendriks J, Hindricks G, Manolis AS, Oldgren J, Popescu BA, Schotten U, Van Putte B, Vardas P. ESC guidelines for the management of atrial fibrillation developed in collaboration with EACTS. Eur Heart J, 2016. 2016;37(38):2893-962. https://doi.org/10.1093/eurheartj/ ehw210.

2. January CT, Wann LS, Calkins H, Chen LY, Cigarroa JE, Cleveland JC Jr, Ellinor PT, Ezekowitz MD, Field ME, Furie KL, Heidenreich PA, Murray KT, Shea JB, Tracy CM, Yancy CW. 2019 AHA/ACC/HRS Focused Update of the 2014 AHA/ACC/HRS Guideline for the Management of Patients With Atrial Fibrillation: A Report of the American College of Cardiology/American Heart Association Task Force on Clinical Practice Guidelines and the Heart Rhythm Society. J Am Coll Cardiol. 2019;74(1):104-32. https://doi.org/10.1016/j.jacc. 2019.01.011
3. Lip GY. The prothrombotic state in atrial fibrillation: new insights, more questions, and clear answers needed. Am Heart J. 2000;140(3):348-50. https://doi.org/10.1067/mhj.2000.108521.

4. Turgut O, Zorlu A, Kilicli F, Cinar Z, Yucel H, Tandogan I, Dokmetas HS. Atrial fibrillation is associated with increased mean platelet volume in patients with type 2 diabetes mellitus. Platelets. 2013;24(6):493-7. https://doi.org/10. 3109/09537104.2012.725876.

5. Pafili K, Penlioglou T, Mikhailidis DP, Papanas N. Mean platelet volume and coronary artery disease. Curr Opin Cardiol. 2019;34(4):390-8. https://doi.org/ 10.1097/hco.0000000000000624.

6. Braekkan SK, Mathiesen EB, Njolstad I, Wilsgaard T, Stormer J, Hansen JB. Mean platelet volume is a risk factor for venous thromboembolism: the Tromso study, Tromso, Norway. J Thromb Haemost. 2010;8(1):157-62. https://doi.org/10.1111/j.1538-7836.2009.03498.x.

7. Hong SP, Choi DH, Kim HW, Kim BB, Chung JW, Koh YY, Chang KS. Stroke prevention in patients with non-valvular atrial fibrillation: new insight in selection of rhythm or rate control therapy and impact of mean platelet volume. Curr Pharm Des. 2013;19(32):5824-9.

8. Bayar N, Arslan S, Cagirci G, Ureyen CM, Cay S, Yuksel IO, Koklu E, Erkal Z, Kucukseymen S. Usefulness of mean platelet volume for predicting stroke risk in paroxysmal atrial fibrillation patients. Blood Coagul Fibrinolysis. 2015; 26(6):669-72. https://doi.org/10.1097/mbc.0000000000000334.

9. Ha SI, Choi DH, Ki YJ, Yang JS, Park G, Chung JW, Koh YY, Chang KS, Hong SP. Stroke prediction using mean platelet volume in patients with atrial fibrillation. Platelets. 2011;22(6):408-14. https://doi.org/10.3109/09537104. 2011.560306.

10. Choi DH, Kang $\mathrm{SH}$, Song $\mathrm{H}$. Mean platelet volume: a potential biomarker of the risk and prognosis of heart disease. Korean J Intern Med. 2016;31(6): 1009-17. https://doi.org/10.3904/kjim.2016.078

11. Chinese Academy Of Neurology. Guidelines for the diagnosis and treatment of acute ischemic stroke in China in 2014. Chinese J Neuro. 2015;4(48)24657.

12. Cetin M, Bakirci EM, Baysal E, Tasolar H, Balli M, Cakici M, Abus S, Akturk E, Ozgul S. Increased platelet distribution width is associated with ST-segment elevation myocardial infarction and thrombolysis failure. Angiology. 2014; 65(8):737-43. https://doi.org/10.1177/0003319713520068.

13. Wan ZF, Zhou D, Xue JH, Wu Y, Wang H, Zhao Y, Zhu L, Yuan ZY. Combination of mean platelet volume and the GRACE risk score better predicts future cardiovascular events in patients with acute coronary syndrome. Platelets. 2014;25(6):447-51. https://doi.org/10.3109/09537104 2013.830708.

14. Li B, Liu X, Cao ZG, Li Y, Liu TM, Wang RT. Elevated mean platelet volume is associated with silent cerebral infarction. Intern Med J. 2014;44(7):653-7. https://doi.org/10.1111/imj.12454.

15. Korniluk A, Koper-Lenkiewicz OM, Kaminska J, Kemona H, Dymicka-Piekarska $\checkmark$. Mean platelet volume (MPV): new perspectives for an old marker in the course and prognosis of inflammatory conditions. Mediat Inflamm. 2019; 2019:9213074. https://doi.org/10.1155/2019/9213074.

16. Koupenova M, Kehrel BE, Corkrey HA, Freedman JE. Thrombosis and platelets: an update. Eur Heart J. 2017;38(11):785-91. https://doi.org/10.1093/ eurheartj/ehw550

17. Choi SW, Kim BB, Choi DH, Park G, Shin BC, Song H, Kim D, Kim DM. Stroke or left atrial thrombus prediction using antithrombin III and mean platelet volume in patients with nonvalvular atrial fibrillation. Clin Cardiol. 2017; 40(11):1013-9. https://doi.org/10.1002/clc.22759.

\section{Publisher's Note}

Springer Nature remains neutral with regard to jurisdictional claims in published maps and institutional affiliations. 